\title{
PHOTODYNAMIC THERAPY FOR ENDOBRONCHIAL MALIGNANT DISEASE: A PROSPECTIVE FOURTEEN-YEAR STUDY
}

James S. McCaughan, Jr., MD

Thomas E. Williams, MD

\begin{abstract}
Background: After intravenous injection, the photosensitizer dihematoporphyrin ether is selectively retained in tumor cells. This photosensitizer absorbs $630 \mathrm{~nm}$ wavelength light energy and produces a singlet oxygen that destroys the tumor. Photodynamic therapy was performed on endobronchial tumors with the use of light generated by an argon dye laser system delivered through cylinder diffusing tip quartz fibers passed through the biopsy channel of a flexible endoscope. Objectives: Our objectives were to determine factors affecting survivals, benefits, and complications. Methods: From 1982 to May 1996, photodynamic therapy was performed on 175 patients with endobronchial tumors. Sixteen had stage I disease, 9 stage II, 42 stage IILA, 64 stage IIIB, and 44 stage IV. All were followed up until death or November 1996. Results: Multivariate analysis of survival of the effects of age, sex, race, histologic features, Karnofsky Performance Status, and clinical stage showed the clinical stage $(p<0.0001)$ to be the most statistically significant factor. Sixteen patients with stage I disease had a 93\% 5-year disease-related estimated survival. Median (months) survivals were as follows: stage $I=$ not reached; stage $I I=22.5$; stage IIIA $=5.7$; stage IIIB $=55$; and stage IV $=5.0$. Performance status does become significant when it reaches 50 but was not significant for stages I or II. Conclusions: Photodynamic therapy may be considered as an alternative treatment for patients under consideration for surgical treatment for stage I carcinoma in whom the risk of surgery is high. The length of palliation for patients with noncurative disease was equal to or better than that reported historically for most other treatment regimens. (J Thorac Cardiovasc Surg 1997;114:940-7)
\end{abstract}

T he use of selective photodynamic therapy (PDT) to treat patients with malignant tumors is based on three observations: (1) after being injected intravenously, the photosensitizer disseminates to all cells; (2) owing to differences in vascular and lymphatic clearance from tumors and retention of the photosensitizer by the tumor cells, the photosensitizer is selectively retained in the tumor cells and interstitial tissue of the tumor, so that after 2 days

From the Grant Laser Center, Columbus, Ohio.

Read at the Seventy-seventh Annual Meeting of The American Association for Thoracic Surgery, Washington, D.C., May 4-7, 1997.

Received for publication May 5, 1997; revisions requested July 11, 1997; revisions received July 31, 1997; accepted for publication July 31, 1997.

Address for reprints: James S. McCaughan, Jr., MD, FACS, FCCP, 323 E. Town St., Columbus, OH 43215.

Copyright (C) 1997 by Mosby-Year Book, Inc.

$0022-5223 / 97 \$ 5.00+0 \quad \mathbf{1 2 / 6 / 8 5 2 7 5}$ there is a greater concentration of the photosensitizer in the tumors than in the adjacent normal tissue; (3) the photosensitizer will absorb light energy and produce singlet oxygen, which destroys the tumor. Because there is less photosensitizer in the adjacent tissue, it will react less. We report here our experience using PDT to treat patients with primary endobronchial and tracheal adenocarcinoma and squamous cancer.

A limiting factor in the effectiveness of PDT as currently performed is the fact that the light penetrates only 5 to $10 \mathrm{~mm}$. The bronchi, however, have a maximum diameter of $10 \mathrm{~mm}$ and therefore PDT is ideally suited to relieving obstruction resulting from endobronchial tumors.

\section{Patients and methods}

In a prospective study from 1982 to May 1996, we used PDT to treat 175 patients with primary endobronchial or tracheal carcinoma and evaluated their survival up to 
Volume 114, Number 6

November 1996. Entry criteria were a histologic diagnosis of adenocarcinoma or squamous cell carcinoma and failure of conventional treatment, ineligibility for them because of medical status, or patient refusal. Follow-up was $100 \%$. One hundred seven patients were white men, 52 white women, 12 black men, three black women, and one Hispanic woman. Seventeen had adenocarcinoma and 158 squamous carcinoma. Their ages ranged from 35 to 97 years with a mean and median of 65 years. One hundred twenty-eight had had unsuccessful conventional therapies (chemotherapy and/or surgery, and/or external radiation or brachyradiation, and/or yttrium-aluminum-garnet [YAG] laser). Forty-seven had no previous treatment.

All patients signed an informed consent form as part of a protocol approved by the Institutional Review Board of the Grant Laser Center, and their disease was clinically staged at the time of the PDT according to the TNM system ${ }^{1}$ based on the history, physical examination, bronchoscopic findings, and computed tomographic scans of the chest and abdomen. Sixteen patients had stage I disease (all squamous), 9 stage $\Pi$ (all squamous), 42 stage IIIA ( 3 adenocarcinoma, 39 squamous), 64 stage IIIB (7 adenocarcinoma, 57 squamous), and 44 had stage IV disease ( 7 adenocarcinoma, 37 squamous).

Three other patients with squamous cell carcinoma-insitu of the endobronchial tree were also treated.

PDT was performed with $630 \mathrm{~nm}$ light generated by an argon dye laser system (Spectra Physics, Mountain View, Calif.) as the activator delivered through cylinder diffusing tip quartz fibers passed through the biopsy channel of a flexible endoscope. These 0.5 to $2.5 \mathrm{~cm}$ diffuser tips are modified to deliver the light 360 degrees radially. When possible, the diffuser tip was inserted into the tumor (interstitial treatment). However, it usually was placed alongside the tumor (surface treatment).

Initial treatments were performed with hematoporphyrin derivative as the photosensitizer, but for the past 12 years we have used the more purified form dihematoporphyrin ether (DHE) (Quadra Logic Technologies, Vancouver, B.C., Canada) injected intravenously.

Various photosensitizer doses, day of treatment after injection, light power densities, and light doses were evaluated. ${ }^{2-4}$ Analyses of the results from these various parameters led us to now use $60 \mathrm{mg}$ of DHE per meter squared of body surface. Initially PDT was performed 2 days after the injection, but for the past 7 years we have been treating patients on the same day as the injection, injecting in the morning and doing the bronchoscopic treatment and PDT in the afternoon. PDT can be done as soon as 10 minutes or as late as 7 days after injection of the sensitizer. ${ }^{5}$ At the end of the treatment we mechanically remove as much of the tumor as possible with forceps and snares. This is an integral part of the treatment for obstructive lesions but not for superficial lesions. After PDT the tumors can be removed mechanically without fear of excessive bleeding. Fogarty catheters and arterial balloons are used to dilate bronchi and remove tumor and any blood clots if needed.

For several years we used power densities of $500 \mathrm{~mW}$ per centimeter of diffusing fiber ( $\mathrm{mW} / \mathrm{CF}$ ); however, manufacturer's modifications of the fibers now cause them to burn off at this power. Therefore we now use 400
$\mathrm{mW} / \mathrm{CF}$. Light doses are 400 joules per centimeter of diffusing fiber $(\mathrm{J} / \mathrm{CF})$ for trachea and main bronchi; 300 $\mathrm{J} / \mathrm{CF}$ for lobar bronchi, and $200 \mathrm{~J} / \mathrm{CF}$ for segmental bronchi.

Toilet bronchoscopic treatments are done 1 day and 3 days after PDT. These are essential treatments and must be done to remove exudate and secretions. If residual viable tumor is found at the first toilet bronchoscopy we retreat this site. This can usually be determined clinically by blood oozing from the sites after tumor removal. The reacted tumor does not bleed.

We now use light general anesthesia almost exclusively for the bronchoscopic treatment, regardless of the patient's respiratory status. It is important to keep the anesthetist aware of how much time will be needed for the procedure to permit extubation at the end of the bronchoscopic treatment or in the recovery room. We do not use narcotics. The arterial oxygen saturation and expired carbon dioxide level are continuously monitored, and during the treatment the inspired oxygen fraction is kept below $40 \%$. If the patient's oxygen saturation falls below $90 \%$ we stop the treatment, ventilate with $100 \%$ oxygen until adequate saturation is obtained, and then drop the inspired oxygen fraction to $40 \%$ and continue the treatment. The standard flexible bronchoscope, lubricated with silicone, is passed through an $8.5 \mathrm{~mm}$ endotracheal tube. We have not used a rigid bronchoscope for 10 years.

One month after PDT a repeat endoscopic examination was done, and residual tumor was treated after another injection of photosensitizer. Patients were then periodically subjected to endoscopic examination and treated again for symptomatic residual tumor. Two hundred ninety-five injections of photosensitizer were made (mean 1.7 per patient) and 492 PDT bronchoscopic treatments were performed (mean 2.8 per patient). Eleven hundred twenty-eight treatments were done to 673 different sites.

The clinical stage of disease, Karnofsky Performance Status (KPS), ${ }^{6}$ weight, diet, and ordinal scaled levels of pain, dyspnea, oxygen requirement, hemoptysis, and cough were recorded at each endoscopic examination. Biopsy specimens and brushings were taken at each endoscopic study. At the beginning and end of each bronchoscopic treatment the minimal diameter open and the percent of obstruction were estimated by using biopsy forceps, the known diameter of the bronchoscope, and photographs. Complications, the color and consistency of the tumor, and the amount of edema, exudate, bleeding, and mucositis were evaluated and recorded by means of ordinal scales.

The survival status or time and cause of death were verified by direct communication with the patient or family and the primary physician.

No patients were refused treatment because of a low KPS. Frequently their poor status was due to an obstructive lesion and if it could be removed their condition could be improved.

Statistical analyses were performed with the use of SuperANOVA, StatView, and Survival Tools (Abacus Concepts, Inc., Berkeley, Calif.). Disease-specific survival times in months were calculated from the time of their first treatment to the end point of November 1996 by means of Kaplan-Meier tables and curves. The Breslow- 


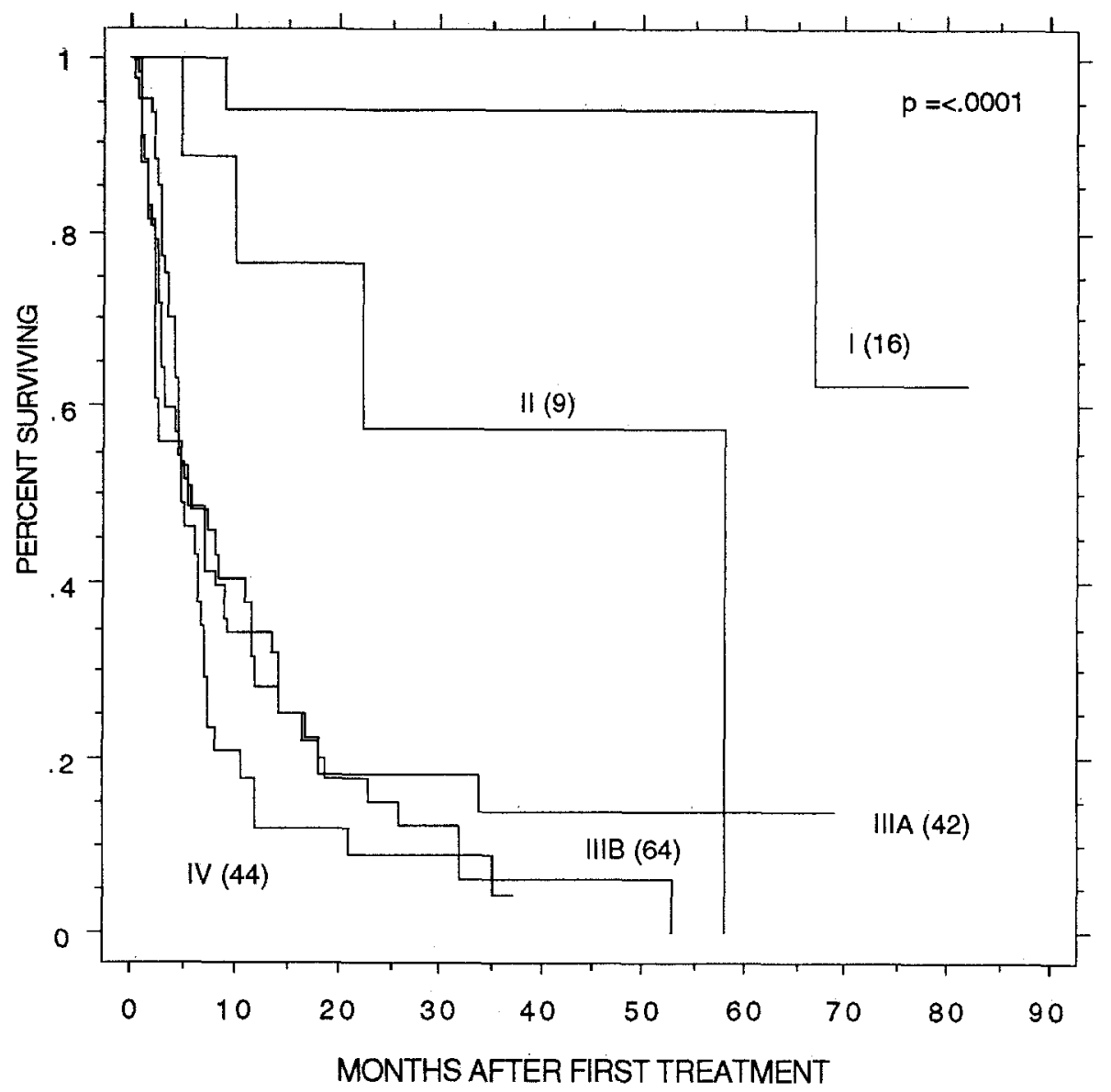

Fig. 1. Kaplan-Meier cumulative survival curves for patients with endobronchial carcinoma treated with PDT stratified by their clinical stage at the time of PDT.

Gehan-Wilcoxon test was used to compare the significance of differences of survival distributions because it is more likely to detect early differences than log-rank tests and most of the deaths occur early in all series of endobronchial cancer. Cox proportional hazards tests were used to estimate the effects of different variables on the length of survival. All statistics used $95 \%$ confidence limits.

\section{Results}

The effect of different variables on survival of 175 patients with adenocarcinoma or squamous carcinoma after PDT was estimated by means of multivariate analysis. A model of the effects of age $(p=0.599)$, sex $(p=0.923)$, race $(p=0.903), \operatorname{KPS}(p=0.013)$, histologic characteristics $(p=0.301)$, and clinical stage showed that the most statistically significant variable was the stage ( $p=0.0001)$. The global likelihood ratio for the model was significant $(p<0.0001)$.

The median survival of all patients was 7 months (mean $=18.6$ months $)$. Median survivals stratified by clinical stage in months were as follows: stage I = not reached; stage II $=22.5$; stage IIIA $=5.7$; stage IIIB $=5.5$, and stage IV $=5$. Mean survivals stratified by clinical stage were as follows: stage $\mathrm{I}=$ 63 ; stage II $=39$; stage IIIA $=11$; stage IIIB $=12$, and stage IV $=8$.

When split by KPS, there is a significant difference in the survival, but the KPS does not become significant until it falls below 50 and is not significant for stages I or II. For stage IIIA the median survival was 8.2 months when the KPS was greater than 50 and 2.0 for a KPS less than 50. For stage IIIB the median survival was 7.2 months when the KPS was more than 50 and 4.0 for a KPS less than 50 . For stage IV survival was 6.5 months when the KPS was greater than 50 and 2.6 for a KPS less than 50 .

Thirty-two ( $21 \%$ ) of 150 patients with stage III or IV disease survived 12 months or more, and six of 
Volume 114, Number 6

Table I. Patients who died within 30 days of first treatment

\begin{tabular}{|c|c|c|c|c|c|c|c|c|c|c|}
\hline Cause of death & $\begin{array}{l}\text { Hernoptysis } \\
\text { grade }\end{array}$ & $\begin{array}{l}\text { Oxygen } \\
\text { requirement } \\
\text { grade }\end{array}$ & $\begin{array}{c}\text { Dyspnea } \\
\text { level } \\
\text { grade }\end{array}$ & $K P S$ & Stage & Histology & $\begin{array}{l}\text { Age } \\
(y r)\end{array}$ & Sex & Race & $\begin{array}{c}\text { Months } \\
\text { after } \\
\text { treatment }\end{array}$ \\
\hline Pulmonary hemorrhage & 2 & 1 & 2 & 60 & IIIA & SQ & 55 & M & W & 0.8 \\
\hline Pulmonary hemorrhage & 2 & 2 & 4 & 30 & IIIB & SQ & 75 & M & W & 0.8 \\
\hline $\begin{array}{l}\text { Pulmonary hemor- } \\
\text { rhage, abscess cavity }\end{array}$ & 3 & 2 & 2 & 50 & IIIB & SQ & 75 & M & B & 0.5 \\
\hline Pulmonary hemorrhage & 3 & 0 & 2 & 70 & IV & SQ & 62 & M & B & 0.8 \\
\hline $\begin{array}{l}\text { Stroke, respirator, ex- } \\
\text { tubated per family }\end{array}$ & 0 & 0 & 1 & 70 & IIIB & $\mathrm{SQ}$ & 76 & M & W & 0.8 \\
\hline $\begin{array}{l}\text { Pneumonia, renal fail- } \\
\text { ure sepsis }\end{array}$ & 0 & 2 & 4 & 40 & IIIA & SQ & 70 & M & W & 0.5 \\
\hline $\begin{array}{l}\text { Pneumonia, respirator, } \\
\text { extubated per family }\end{array}$ & 0 & 2 & 4 & 30 & IIIA & SQ & 60 & M & W & 0.3 \\
\hline Dead of disease & 0 & 2 & 4 & 20 & IIIA & $\mathrm{AD}$ & 60 & $\mathrm{~F}$ & W & 0.8 \\
\hline
\end{tabular}

these had a KPS less than 50. Patients who survived more than 12 months are listed by clinical stage:

Stage IIIA: 12, 14, 16, 17, 18, 27, 34, 35, 53, 69 months

Stage IIIB: 13, 14(4), 15, 17, 18, 19, 23, 26, 28, 29, 30, 32,53 months

Stage IV: 12(2), 21, 24, 35, 37 months

When clinical stages IIIA, IIIB, and IV were analyzed as a separate subgroup, the multivariate analysis showed the KPS $(p=0.0102)$ statistically more significant than the stage $(p=0.3071)$. Also, Breslow-Gehan-Wilcoxon analysis of Kaplan-Meier survival curves for the subgroup stages IIIA, IIIB, and IV was significant for grouping by KPS ( $p=$ 0.0001 ) but not significant for grouping by clinical stage $(p=0.292)$. Fig. 1 shows the Kaplan-Meier survival estimates for patients with endobronchial tumor stratified by clinical stage.

Three patients with squamous cell carcinoma in situ had complete responses with no evidence of recurrence at 8,74 , and 121 months.

Sixteen patients had clinical stage I disease. All were deemed medically ineligible for surgery by thoracic surgeons. The estimated disease-related 5-year survival for all patients with stage I disease was $93 \%$. Eleven are still alive with no evidence of disease a median of 29 months (mean 37 months) after their first treatment. Two died of their disease (one of pulmonary hemorrhage at 9 months and one of brain metastases at 67 months). Three other patients died of unrelated diseases (one at 11.7 months of emphysema and congestive heart failure, one at 12.5 months of a myocardial infarction, and one of a stroke at 27.5 months).
Complications. There were no intraoperative deaths during PDT.

Eight patients died within 30 days of their first PDT treatment (4\%). Four had stage IIIA disease, three stage IIIB, and one stage IV. Their median KPS was 45 (mean 46) and median age was 65 years (mean 67 years). The causes and time of these deaths and their pretreatment conditions are listed in Table I.

While we were delivering oxygen down the fiberoptic bronchoscope during a treatment with a cylinder fiber in one of our first patients, the fiber broke and the oxygen ignited the end of the bronchoscope. The patient had no serious consequences. Since then we have maintained the inspired oxygen fraction below $40 \%$ during the treatment. PDT causes an edematous and exudative reaction, which may completely obstruct a major airway. One patient treated by insertion of a straight-tip fiber into a retrotracheal tumor invading the trachea had a tracheoesophageal fistula 1 week after PDT. After repeated treatments, several patients had strictures completely obstructing bronchi with scar tissue that showed no evidence of tumor on biopsy, brushing, and washings for cytologic examination. Solar photosensitivity of the skin may last for up to 8 weeks after the injection of DHE.

\section{Discussion}

From treating and monitoring these patients with a bronchoscope, it became obvious that many patients die and suffer, not because they have lung cancer in the parenchyma, lymph nodes, or extrathoracic metastases, but because of the obstruction of bronchi or contiguous bronchi leading to lung tissue 
Table II. Lung cancer median survival in months

\begin{tabular}{ccccccc}
\hline Clinical stage & Mountain $^{8}(S)$ & Bulzebruck $^{9}(S)$ & Rosenthal $^{10} .(X)$ & $P D T$ & $P D T(K P S \geq 50)$ & $P D T(K P S<50)$ \\
\hline I & 48 & 38 & 19 & Not reached & Not reached & NA \\
II & 18 & 17 & 18 & 22.5 & 22.5 & NA \\
IIIA & 12 & 12 & 12 & 6 & 8 & 2 \\
IIIB & 7 & 8 & 10 & 6 & 7 & 4 \\
IV & 4 & 6 & - & 5 & 7 & 3 \\
\hline
\end{tabular}

$S$, Surgery; $X$, x-ray therapy; $N A$, not applicable.

that does not have cancer in it per se, or by bleeding or sepsis or a combination of these. We strongly disagree with investigators who have not used laser therapy when they have found the lesion was "too small" or the patient did not have symptoms. If anything, we have regretted not readministering bronchoscopic treatments sooner before the tumor spread to other bronchi or became large and bloody. The intraoperative and perioperative risk of complications of therapy are much greater for the patient who has almost complete obstruction of bronchi on which he is dependent for aeration than when the bronchi are not as obstructed or the tumor is confined to one bronchus. As tumors enlarge, so does their blood supply, as well as the length of time required for the endoscopic treatment. It is better to treat patients when the lesion is small and every few months than to wait until the patient is dying.

Also, there is a benefit to delaying the central endobronchial spread of the tumor. The patient should be treated even if the tumor is large and the distal bronchus cannot be opened. Although the tumor obstructing the bronchus may be only a small part of the tumor, frequently this is causing the symptoms. Patients have maintained a KPS of 90 for 5 years with repeated bronchoscopic PDT.

The most significant variable affecting the length of survival in our series was the stage of the disease. When staged, there was no statistically significant difference related to age, sex, or race.

There was no statistically significance difference in the survival distributions within each stage between adenocarcinoma and squamous cell carcinoma. However, within stages IIIA, IIIB, and IV, a KPS above or equal to 50 was a significant favorable prognosticator and should be considered in comparing survivals from different treatment regimens for these stages. The significance of KPS on survival of the individual patient, however, depends on the cause of the decreased performance. If it is due to endobronchial obstruction causing dyspnea, bleeding, or pneumonia, the condition of these patients can be greatly improved by removing the obstruction or stopping the bleeding. Patients with a KPS of 30 survived 16 (IIIA), 32 (IIIB), 35 (IIIA), and 37 (IV) months. Two patients with a KPS of 40 survived 12 (IV) and 17 (IIIB) months.

Comparison of survival after PDT with other treatment regimens. Flehinger, Kimmel, and Melamed $^{7}$ evaluated the survival of patients with clinical stage I non-small-cell lung cancer who refused or were considered medically ineligible for surgical treatment $(n=92)$ compared with those who were treated surgically $(n=434)$. The 5 -year cumulative survival probability after surgery was $62 \%$ at SloanKettering, 53\% at Johns Hopkins, and 52\% at the Mayo Clinic, and the 5-year survival probability for patients not treated surgically was $0 \%$ to $7 \%$. The 5-year disease-related survival after PDT for 16 patients with stage I carcinoma of the endobronchus is estimated to be $93 \%$. Table II compares the median survival after PDT by stage with the survival after surgical therapy, as reported by Mountain ${ }^{8}$ and Bulzebruck and associates, ${ }^{9}$ or irradiation, as reported by Rosenthal and colleagues. ${ }^{10}$

From an analysis of PDT and YAG laser treatment bronchoscopies, we ${ }^{11}$ found no statistically significant difference in the mean time required for the treatments or in the change in the arterial blood $\mathrm{pH}$, carbon dioxide tension, or oxygen tension as measured in the recovery room after treatment. Analysis of the time after treatment required for reobstruction of the bronchus after PDT versus YAG laser showed the YAG laser reduced the amount of obstruction more at the end of treatment than PDT. However, after toilet bronchoscopy of the PDT-treated sites, the degree of obstruction was essentially the same, and the sites treated with the YAG laser became reobstructed faster than the PDT-treated sites when compared 2 and 4 weeks after treatment. ${ }^{12}$

The fiber for PDT can be inserted blindly into the tumor for treatment without fear of burning a hole into an adjacent vessel. Endobronchial smoke is 
always produced with the YAG laser, but not with PDT. We have never been able to get a complete response using the YAG laser alone.

Post-PDT pulmonary hemorrhage. Four of the 178 patients died of pulmonary hemorrhage within 30 days of PDT. All had hemoptysis with clots before treatment. Balchum and Doiron ${ }^{13}$ reported that four of 35 patients with endobronchial tumor and severe hemoptysis before treatment died of pulmonary hemorrhage 4 to 5 weeks after PDT. Other techniques for management of endobronchial tumors are also followed by posttreatment death from pulmonary hemorrhage: after brachytherapy, four of 56 patients as reported by Macha and associates ${ }^{14}$ and seven of 18 patients as reported by Marsh $^{15}$; after YAG laser: three of 27 patients as reported by Gelb and Epstein ${ }^{16}$ and three of 30 patients as reported by Brutinel and colleagues. ${ }^{17}$ This complication may be related to the natural history of the disease. Many patients are referred to us for the problem of previous massive pulmonary bleeding.

Photosensitizer dosage: milligrams per kilogram or milligrams per square meter of body surface? The effect of different variables on the percentage decrease of primary endobronchial obstruction obtained with PDT was evaluated in 195 treatments. ${ }^{18}$ There were no statistically significant differences in the percent decrease of obstruction of the bronchus when $1,1.5$, or $2 \mathrm{mg} / \mathrm{kg}$ were used as the parameters. However, when the quartiles of milligrams per square meter were used as parameters, there was a significant difference between the results obtained, comparing the low with the inner quartiles $(p=$ $0.0433)$ and the inner with the high quartiles $(p=$ $0.0007)$. The regression curve for the effect of the milligrams per square meter showed a peak decrease in the amount of obstruction at $60 \mathrm{mg} / \mathrm{m}^{2}$ (a mean of $1.4 \mathrm{mg} / \mathrm{kg}$ ). This is the drug dose we now use.

Indications for PDT. PDT is ideally suited to relieving endobronchial obstructions caused by any tumors. All histologic types of endobronchial tumor (primary or metastatic) responded to PDT. PDT can improve or delay progression of tumor and symptoms such as bleeding, secretions, and dyspnea and thereby improve the quality of life and increase survival time. Many patients die of their local disease long before they would have died of metastases. Survivals of patients with carcinoma-in-situ and stage I disease appear to be as good as those historically reported for surgical treatment. Com- plete responses of Tis and stage I endobronchial cancers treated with only one PDT have lasted up to 7 years. Randomized clinical trials should be used to compare the effectiveness of PDT versus surgical therapy in early-stage lung cancer or to compare PDT versus chemotherapy and radiation for patients who are ineligible for surgery.

Hayata and colleagues ${ }^{19}$ have preoperatively treated patients with PDT to convert them from inoperable to operable candidates. We treated a patient with bilateral synchronous tumors by first performing a right upper lobectomy for the larger adenocarcinoma. Several months later, we treated the squamous carcinoma-in-situ in the left upper lobe with PDT. If PDT had not been available, the operation would not have been done because his pulmonary function would not permit bilateral resections. He has no evidence of disease 121 months after PDT.

Recurrences in bronchial stumps are excellent tumors to treat with PDT because there is little chance for complication from exudate. Historically, the mean survival for patients with bronchial stump recurrence is 9 months. ${ }^{20}$ We have been able to keep some of these patients alive and functioning for 5 years by repeated bronchoscopic treatments and treatments of recurring disease. Often, our goal cannot be to improve the patient's condition, but only to delay its deterioration. PDT enables destruction of the invisible submucosal tumors that will grow proximally and obstruct other bronchi. Debulking and decreasing tumor burden before irradiation or chemotherapy can improve respiratory function sooner.

PDT causes thrombosis of small vessels and often can be used to control bleeding from them regardless of their location or cause. The scaled amount of bleeding was recorded before, during, and at the end of PDT at 283 consecutive bronchoscopic PDTs. According to Student's $t$ test, there was a significant decrease in the bleeding during and at the end of PDT compared with before PDT $(p<0.001)$. We used PDT to treat three patients with uncontrollable class 3 or 4 hemoptysis from nonmalignant lesions. There has been no recurrence of hemoptysis at 5, 13, and 17 months after PDT. ${ }^{21}$ PDT stopped lifethreatening hemoptysis in a patient with hereditary telangiectasia after conventional therapies failed. He had no bleeding for 26 months after his first treatment and has had no bleeding for 41 months after a second PDT treatment for recurrence of bleeding. ${ }^{22}$ 


\section{REFERENCES}

1. Beahrs OH, Henson D, Hutter RVP, Kennedy BJ, editors. Manual for staging of cancer. 4th ed. American Joint Committee on Cancer. Philadelphia: JB Lippincott; 1992.

2. McCaughan JS, Hawley PC, Bethel BH, Walker J. Photodynamic therapy of endobronchial malignancies. Cancer 1988; 62:691-701.

3. McCaughan JS Jr, Barabash R, Hawley P. Stage III endobronchial squamous cell cancer: survival after Nd:YAG laser combined with photodynamic therapy vs. Nd:YAG laser or photodynamic therapy alone. The Society of Photo-Optical Instrumentation Engineers 1991;1426:279-86.

4. McCaughan JS Jr. Photodynamic therapy of malignancies: a clinical manual. Austin (TX). RG Landes; 1992.

5. McCaughan JS Jr. Effect of photodynamic therapy on same day of injection of dihematoporphyrin ether on endobronchial tumors (abstract). Lasers Surg Med Suppl 1992;4:49.

6. Karnofsky DA, Burchenal JH. The clinical evaluation of chemotherapeutic agents in cancer. In: Macleod CM, editor. Evaluation of chemotherapeutic agents. New York: Columbia University Press; 1949. p. 199-205.

7. Flehinger BJ, Kimmel M, Melamed MR. The effect of surgical treatment on survival from early lung cancer. Chest 1992;101:1013-8.

8. Mountain C. Value of the new TNM staging system for lung cancer. Chest 1989;96:47s-49s.

9. Bulzebruck H, Bopp R, Drings P, Bauer E, Krysa S, Probst $G$, et al. New aspects in the staging of lung cancer. Cancer 1992;70:1102-10.

10. Rosenthal SA, Curran WJ Jr, Herbert SH, Hughes EN, Sandler HM, Stafford PM, et al. Clinical stage II non-small cell lung cancer treated with radiation therapy alone. Cancer 1992;70:2410-7.

11. McCaughan JS Jr, Barabash RD, Penn GM, Glavan BJ. $\mathrm{Nd}: Y A G$ laser and photodynamic therapy for esophageal and endobronchial tumors under general and local anesthesia: effects on arterial blood gas levels. Chest 1990;98:1374-8.

12. McCaughan JS Jr. Photodynamic therapy versus ND-YAG laser treatment of endobronchial or esophageal malignancies. In: Spinelli P, Dal Fante M, Marchesini R, editors. Photodynamic therapy and biomedical lasers. New York: Elsevier; 1992. p. 23-36.

13. Balchum OJ, Doiron DR. Photoradiation therapy of endobronchial lung cancer. In: Unger M, editor. Clinics in chest medicine. Philadelphia: WB Saunders; 1985. p. 255-75.

14. Macha HN, Koch K, Stadler M, Schumacher W, Krumhaar D. New technique for treating occlusive and stenosing tumors of the trachea and main bronchi: endobronchial irradiation with high-dose iridium-192 combined with laser canalization. Thorax 1987;42:511-5.

15. Marsh BR. Bronchoscopic brachytherapy. Laryngoscope 1989;99:1-13.

16. Gelb AF, Epstein JD. Laser in treatment of lung cancer. Chest 1984;86:662-6.

17. Brutinel WM, Cortese DA, McDougall JC, Gillio RG, Bergstrahl EJ. A two-year experience with the neodymium-YAG laser in endobronchial obstruction. Chest 1987;91:159-65.

18. McCaughan JS Jr, Miller C. Photosensitizer dosage: $\mathrm{mg} / \mathrm{kg}$ or mg/2? In: Dougherty TJ, editor. Optical methods for tumor treatment and detection: mechanisms and techniques in photodynamic therapy II. The Society of Photo-Optical Instrumentation Engineers Proceedings. 1993;1881:26-34.
19. Hayata Y, Kato H, Konaka C, Ono J, Takizawa N. Hematoporphyrin derivative and laser photoradiation in the treatment of lung cancer. Chest 1982;81:269-77.

20. Kaiser LR, Keller SM, Fleshner P. The significance of microscopic residual disease at the bronchial resection margin (abstract). Fifty-fourth annual meeting of the American College of Chest Physicians. 6 Oct 1988. p. 848.

21. McCaughan JS Jr, Williams TE Jr, Hawley PC, Miller C. Endobronchial photodynamic therapy for hemoptysis. In: Dougherty T, editor. The Society of Photo-Optical Instrumentation Engineers Proceedings: Optical methods for tumor treatment and detection. 1994:2113:2-9.

22. McCaughan JS Jr, Hawley PC, LaRosa JC, Thomas JH, Hicks WJ. Photodynamic therapy to control life threatening hemorrhage from hereditary hemorrhagic telangiectasia. Lasers Surg Med 1996;19:492-4.

\section{Discussion}

Dr. Douglas E. Wood (Seattle, Wash.). I congratulate the authors on their superb results using endobronchial PDT for primary endobronchial and tracheal carcinoma. This series of 175 patients is the largest series in the United States and rivals the Japanese experience in this arena.

The authors' conclusions that survival is based on tumor stage and functional status are self-evident. Actual 5-year survival, however, is the standard used in the oncology literature and is $69 \%$ for stage I disease in this series rather than the $93 \%$ disease-related survival reported. Five-year survival was $0 \%$ in patients with stage II disease, $18 \%$ in those with stage IIIA, and $0 \%$ in both stage IIIB and stage IV disease. Clinical staging with computed tomographic scanning has a sensitivity and specificity of only $65 \%$. Therefore, how confident are you that a significant number of these patients were not both overstaged and understaged, potentially confounding not only your decision-making regarding operability but also your reported survival statistics? What were the reasons for inoperability in patients with stage I disease? Would bronchoplastic resections have been possible if a pneumonectomy was not feasible? Were patients with clinical stage IIIA disease denied consideration for operation on the basis of enlarged lymph nodes without mediastinoscopy? What is the role for PDT in treating early-stage lung cancers with curative intent? Do your data provide enough phase I/II feasibility information to provide the basis for a phase III trial in one of the national oncology groups?

The compelling point from this presentation is that patients with endobronchial obstruction from advanced cancer still have a median survival of 7 months and deserve aggressive airway palliation to manage dyspnea, cough, and obstructive pneumonia.

The authors report an average of three bronchoscopic treatments per patient under general anesthesia for the application of PDT. These patients also had mechanical debridement and dilatation, similar to the techniques that we have reported in more than 250 procedures for endobronchial obstruction at the University of Washington. What is the additional benefit of PDT over these cheaper and more readily available techniques? What equipment must one purchase and at what cost to provide PDT? 
Finally, there are suggestions in the literature that photoporphyrins may also be a radiosensitizer. Is it safe to radiate these patients after injection with DHE, and could this potentially provide even greater oncologic results with selective radiosensitization of the primary and metastatic tumor?

Dr. Joseph LoCicero III (Boston, Mass.). We owe a debt of gratitude to Dr. McCaughan for his perseverance and persistence with a technique that has really been an orphan procedure for about 15 years. This particular approach, the drug and the therapy, now is approved by the Food and Drug Administration for treatment of esophageal cancer. The drug is now available. The cost for the hospital is $\$ 2000$ per vial. It remains an expensive therapy. Dr. Wood mentioned the cost of the laser to go with this. Right now it runs about $\$ 100,000$. This is not a particularly inexpensive therapy. However, there will be a trial for esophageal cancer, really for dysplasia in Barrett's esophagus, which will begin fairly soon. The drug will also be presented to the Food and Drug Administration for approval for treatment in lung cancer and will be available for that therapy most likely within the year.

I have a question specifically for Dr. McCaughan, who has really been a light in the darkness. Are all of these bronchoscopic procedures performed with the patient under general anesthesia and does the patient need to stay in the hospital? When we compare this technique with our present therapies, is there going to be a cost advantage to this particular type of therapy?

In our early experience using PDT, which is now about 5 years old, we found an increased incidence of problems with lung damage in patients who received radiation therapy afterward. Trying to produce an animal model or even a test-tube model to show that there has been increased sensitivity of these cells has been difficult. Do you have any experience to help us with that approach?

Dr. McCaughan. We treat these patients with $\mathrm{x}$-ray therapy if they have not had it within a week or two after the treatment. We have had no increased problems from the radiotherapy.

With regard to the staging, clinical staging is almost worthless, but it is the best thing we have. It never agrees with pathologic staging. It does supply all the statistics, which can be compared.

The problem with doing survival curves is that these are elderly patients who die of other diseases. If you operated on 100 patients and put them in an airplane a week later and they all crashed and died, you would not say that your survival was 1 week. You have to take into account that these patients have other problems, and that is what the standard Kaplan-Meier statistical curve does, the same as the actuarial curves.

The decision was made by thoracic surgeons who referred the patients to us because they believed that these patients would not tolerate the operation because they already had surgery or their pulmonary functions were too poor. This was their decision. Because they were all in private practice, they were not particularly eager to give away patients. Most of the patients with stage IIIA disease had had chemotherapy and x-ray therapy. Many of them had had surgery. The usual litany is surgery, x-ray therapy, and chemotherapy. If that approach does not work, they are sent for PDT. Then we are asked, "Well, how many did you cure?" Obviously we started with poor-risk patients. If PDT can offer them the possibility of 6 months' survival, it is worth trying.

\section{Notice of correction}

In the April 1997 issue of the Journal, in the article by Benetti and associates titled "Minimally Invasive Aortic Valve Replacement" (1997;113;806-7), an error was made. Ines Benetti was incorrectly identified as having an MD degree. 